Preprint typeset in JHEP style - HYPER VERSION

\title{
Shape and holography: studies of dual operators to giant gravitons.
}

\author{
David Berenstein * \\ School of Natural Sciences, Institute for Advanced Study, Princeton, NJ 08540, USA
}

\begin{abstract}
In this paper we study the conjectured dual operators to a near maximal giant graviton and their open string fluctuations in the large $N$ limit. Using matrix model estimates we show that the spectrum of states near the D-brane operator is consistent with a Fock space of open plus closed string states. We also give an argument that these operators, in spite of having large $\mathrm{R}$ charge of order $N$, are amenable to being studied with standard perturbative techniques, which organize themselves in a $1 / N$ expansion. Also the spectrum of operators dual to massless fluctuations on the D-brane is shown to be protected from weak to strong coupling at leading order, so it is possible to read the shape of the dual operator by understanding how the spherical harmonics of the D-brane fluctuations appear.
\end{abstract}

KEYWORDS: AdS/CFT, D-branes.

*dberens@ias.edu 


\section{Contents}

1. Introduction

2. Fock space of fluctuations on a single giant operator 6

3. One loop anomalous dimensions 17

4. Organizing the $1 / N$ expansion. 19

5. Conclusion 21

A. Combinatorial identities 23

\section{Introduction}

The AdS/CFT correspondence [1] is a remarkable duality between a quantum theory of gravity (a string theory or M-theory) on a particular background and an ordinary quantum conformal field theory in fewer dimensions. The most studied example involves type IIB string theory on $A d S_{5} \times S^{5}$ with $R R$ flux and $\mathcal{N}=4 \mathrm{SYM}$ in four dimensions on the Lorentzian manifold $S^{3} \times R$.

These are examples of holography [2, 3]: it is believed that any theory of quantum gravity is a non-local theory ${ }^{1}$, and that the degrees of freedom of a region of space time are captured completely by the boundary of the region. If this is true, then one has to understand how some notion of locality can appear so that the theory has some classical limit as a theory of gravity on a large space time.

To understand the concept of locality one needs a laboratory for holography where these notions can be made sense of. In this case the $\mathcal{N}=4$ SYM theory dual to IIB strings on $A d S_{5} \times S^{5}$ provides the natural setup to ask these questions.

To have an approximate notion of a point in a theory with quantum gravity, we need a probe that can be associated with such a point. To make the notion of a point concrete at some scale (lets say $\Delta$ ), one needs an object whose fluctuations in position are much shorter than the given scale, and which deform the background metric very little. This gives us a bound $M>\Delta^{-1}$. Also, the gravitational radius of the probe should be smaller than $\Delta$. From here another inequality follows, where $M<f\left(\Delta, M_{p l}\right)$ which depends on the dimension of space time.

\footnotetext{
${ }^{1}$ In a theory of quantum gravity there are no local observables because quantum fluctuations destroy any notion of position at arbitrarily small scales.
} 
Putting those bounds together tells us that $\Delta>1 / M_{p l}$ In general this is the best we can hope for. There should be no notion of approximate classical geometry at the Planck scale. In general, to define approximate locality we should be able to take a classical limit in the following sense: consider a family of metrics for space time which vary only in their scale $R$ (the radius in Planck units). The classical geometry will become a better approximation to the quantum system when the radius of the geometry goes to infinity. In this family one would want a probe which is heavy (lets say of the order of the Planck scale, or whose scale is fixed as we take $R$ to infinity). The position of the heavy probe will give us a notion of classical point in the limit $R \rightarrow \infty$, because the fluctuations of the object will become suppressed with respect to the scale $R$.

If we want to understand locality in a (weakly coupled) string theory, before we reach the Planck scale we get to the string scale, and here classical geometry is replaced by a more quantum version of it: stringy geometry (two dimensional CFT's), but this is still far from giving geometrical concepts at the Planck scale.

Therefore, if we want to understand geometry at the string scale we need a probe that is heavy with respect to the string scale, but which is still tractable. The natural object with these properties is a D-brane. These are non-perturbative objects of string theory, and therefore are heavy. In the weak coupling limit one should consider them as boundary states of the world sheet CFT, and in the large radius limit they become geometric objects (the locus where strings end).

These are the objects one would like to study to define locality at the string scale. However, not all D-branes are the same. Some are unstable, some are extended, some configurations are anomalous (their D-brane charge has nowhere to go).

From the space time point of view, all of this seems standard. However, when we mix the ingredient of holography and ask the same questions in the holographic dual of a gravitational theory, we find that we need all of the above considerations before we can make sense of what it means for a brane to have some position (or if the brane is extended some shape). Even after we decide how to understand these questions we need to be able to trust the calculation on the holographic dual of the theory and match the candidate quantum state on the boundary with a semi-classical object in the geometry.

The SYM theory is tractable only at weak coupling, via perturbation theory. The string theory is tractable if the background is weakly curved in string units, and if the string scale is smaller than the gravitational scale. This regime requires that the dilaton be small everywhere, and that the geometry is very large.

Both statements of tractability are determined by the parameters that define the theory. The parameters of the SYM theory are the gauge group $U(N)$, and the complex gauge coupling $\tau \sim \frac{1}{g_{Y M}^{2}}+i \theta$. On the string theory side the parameters are the closed string coupling $\tau \sim \frac{1}{g_{s}}+i a$ and the radius of the $A d S$ space in string units, $R$. The transformations under $S$-duality identify the two complex couplings with each other.

Quantization of the RR flux on the AdS space produces a number for the radius of the sphere in string units, which is of the order $R^{4} \sim g_{s} N \sim g_{Y M}^{2} N$. This is the 't Hooft coupling of the gauge theory, and it dominates the perturbative expansion of the theory in 
the large $N$ limit $[$ ]. If we want quantities to be calculable using the superstring theory we need to take a limit where the string theory is weakly coupled $g_{s} \rightarrow 0$ or very small, and where the radius $R$ is very large in string units. This implies that we should take $N$ very large. Moreover, the strict limit $R \rightarrow \infty, g_{s} \rightarrow 0$ is the supergravity limit of string theory. This regime of calculability is the strong 't Hooft coupling.

Similarly, for calculability in the SYM theory, we would prefer to have a situation where perturbation theory can be trusted. The perturbation theory of the field theory is controlled by the 't Hooft coupling $g_{Y M}^{2} N$, so the perturbation expansion can be trusted if $g_{Y M}^{2} N$ is small.

The AdS/CFT duality is therefore a strong-weak coupling duality, in the sense that calculability forces us to different (in principle incompatible) corners of the parameters. For many processes it is impossible to extrapolate the dependence on the coupling and we have to trust the duality to do a calculation.

The aspect of this setup that makes checks of duality possible is that the theory is supersymmetric, and there are protected objects (BPS states) for which it is possible to compare results at strong and weak coupling. In general we will define almost BPS states as those for which it is possible to extrapolate between weak and strong coupling.

If one wants to study locality with D-brane probes, then one needs to be able to control the calculation, and one wants to have BPS protected objects. There are again non-perturbative states on the $A d S \times S^{5}$ spacetime which are protected by supersymmetry and correspond to D-brane states. These have been labeled giant gravitons [5], and the simplest ones are given by a $D 3$ brane which wraps a round $S^{3}$ in $S^{5}$ and is spinning on the $S^{5}$. Other configurations have been considered [6, 7]

These are not point-like however. They are extended in the the $S^{5}$ direction, and semiclassically they have a shape. What is the description for these D-branes in the holographic dual? How can we test their shape?

The key to answering these questions is on to how to compare calculations on both sides of the duality: the AdS/CFT dictionary. If we say that two quantum theories are equivalent then we should be able to match the states and operators (correlation functions) between both theories. We should be able also to extrapolate these results between weak and strong coupling.

For $A d S_{5} \times S^{5}$, which has 32 supersymmetries, any massless state in ten dimensions is BPS (they preserve half of the supersymmetries), and it has been possible to match the spectrum of gravitational fluctuations with the spectrum of totally symmetric traceless single trace operators $\operatorname{tr}\left(\phi^{i_{1}} \ldots \phi^{i_{k}}\right)$ and their descendants under the full supersymmetry algebra [8, 9]. Their dimension can be calculated in the free field theory, so one can make a complete match between gravitational states on both theories. Also, one can test the interactions, and there are some protected correlation functions of these operators which are calculable and non-renormalized [10].

Matching the supergravity states alone does not imply that one has a full string theory. The large $N$ limit of 't Hooft for the quantum field theory does suggest a string theory, but it is not tractable for most applications. Miraculously there is a way to find some tractable strings on the $A d S$ geometry. It was understood in [11] that one could take a 
geometric plane wave limit in the holographic dual theory. It turned out that for some special states the perturbation theory in the 't Hooft coupling is parametrically suppressed by the quantum numbers of the state. This parametric suppression made it possible to extrapolate the perturbation theory from weak to strong coupling, and the suppression was tied to the state being almost supersymmetric (almost BPS states).

One can define a parameter which measures the deviation from being BPS for a given state.

$$
\eta=\frac{|\Delta-J|}{J}
$$

where $J$ is one of $R$-charges of the state. This parameter $\eta$ does not necessarily control the perturbation theory, but $\eta=0$ is a BPS state. A state is usually almost BPS if in the geometric limit $\eta$ goes to zero. In the plane wave limit $\eta \sim N^{-1 / 2} \rightarrow 0$, and the BPS states can be matched with graviton multiplets on the plane wave.

Similarly if we consider a BPS brane with some stringy excitations on it, one expects that in the geometric limit the D-brane dominates the mass, charge and back reaction of the geometry, so $\eta \rightarrow 0$. This is even true if we consider also some closed string states in the mix.

The limit therefore is a standard image of D-branes: in the spectrum of states there can be open strings and closed strings. The space of states should be approximated in the perturbative string theory limit by a Fock space of open and closed string states.

Now, because the D-brane is an extended object, in the large radius limit one can consider also small fluctuations of shape for the D-brane. These fluctuations are geometrical, and their spectrum becomes independent of the radius in units of $R$. (The tension of the D-brane will appear suppressing the interactions between these fluctuations.) These fluctuations depend on the shape of the D-brane. To see a shape in the holographic dual we should be able to find not just the D-brane state alone, but also the fluctuations around it. Examples of this notion of shape can be found in various different situations, although in most cases the dual holographic theories are not understood very well. To be more specific, in Matrix theory one could define spherical membranes by certain configurations of matrices [12, 13]. The spherical harmonics could be reproduced by understanding the perturbative spectrum of states on the membrane configuration. However, the spherical membrane itself is unstable for flat space. This can be remedied for wrapped membranes on some 2-sphere [14], and one recovered the right spectrum of states in a large $N$ limit. This effect can also be remedied by introducing a flux which stabilizes the spherical membranes. There is a matrix model for M-theory on a maximally supersymmetric plane wave which has these properties [11]. These spherical membranes are remnants of giant gravitons of $A d S_{4,7} \times S_{7,4}$. One can also find evidence for the existence of five-branes 15] by understanding that some fluctuations on the brane world-volume are protected by supersymmetry [16, 17], and therefore their spectrum can be calculated even when the matrix model is strongly coupled. Another example where one can ask what the shape of branes is, arises from exploring boundary states for WZW CFT's in the large level limit [18]. There one does an overlap with a localized wave function to find the location of the branes. However, this example is strictly understood only on the target space and not on it's holo- 
graphic dual theory: it is an example of how stringy geometry becomes classical in the large radius limit. Finally, the string quantization in the plane wave limit 11] can also be considered as a semi-classical expansion of an extended object (the fundamental string) 19. One can think of this expansion in terms of a purported T-duality along a circle of $S^{5}$. This duality exchanges momentum with winding, and a string with a lot of momentum on the $S^{5}$ could be thought of as a long string (with high winding) on the T-dual circle. This would justify a geometric interpretation of the string as an extended object in the T-dual geometry.

For the giant gravitons in $A d S \times S$, the spectrum of fluctuations has been calculated in [20]. Surprisingly, the quantization of modes does not depend on how big the giant graviton is, and it is compatible with dimensions that could be obtained from free field theory. This fact suggests that these states might be protected [21] in the geometric limit.

Dual operators to giant gravitons on $A d S_{5} \times S^{5}$ have been conjectured in the literature [22]. Operators dual to giant gravitons with excitations (in the plane wave limit) have been conjectured in [23] based on the description of states in [21]. These dual operators are determinant and sub-determinant operators, whose dimension is large (of order $N$ ). For these states, non-planar diagrams dominate over planar diagrams, so expressions with traces mix too much to be useful. The tests that have been performed on these states are mostly of group theoretic nature, in that it has been shown that they have the correct $R$ charge and factorize better than traces of the same dimension [24]. Also, it has been argued that BPS correlation functions with gravitons and a giant graviton produces expansions in topologies with boundaries [25]. These tests are of BPS correlators, and depend just on the free field structure of the theory. However, making a systematic expansion with the methods available in the literature seems hard to do.

Of the tests above, only the last one starts to look like a test that would show that the conjectured dual giant gravitons truly represent D-branes. However, since the calculations are BPS protected, it is hard to understand what is the nature of the results that one is obtaining, and one also has to worry about mixing of states.

The objective of this paper is to make the claim that these operators are giant gravitons as solid as possible: to show that the notion of shape and open string excitations emerges asymptotically in the large $N$ limit. We will also show that the operators in question, describing excited D-branes with some spherical harmonics, have protected dimension in the strict large $N$ limit, at small but finite $g_{s}$ and in the leading approximation. We will also show that the physics of these objects can be organized in a $1 / N$ expansion. To simplify the combinatorial problem, we will limit ourselves to configurations of a single giant graviton with (near) maximal angular momentum on the $S^{5}$.

The technical aspects of this paper are mostly combinatoric: how to organize free field diagrams and the perturbation theory around the D-brane states.

The conjectured dual operators are very similar to baryonic operators. The main difficulty in dealing with baryonic objects in the large $N$ limit is that they are usually not amenable to a standard (diagrammatic) perturbation theory [26], although there are other approximations which can be useful. Perturbative corrections tend to pile up with powers of $N$, just from combinatorial multiplicity of the 'partons'. Since the giant gravitons 
are BPS, there is a possibility that most of these diagrams actually cancel, and that all corrections are due just to the deviation from the object being BPS. We will show that this second intuition turns out to be correct, and that one can organize the perturbative diagrams systematically for these baryonic like-objects. This procedure involves turning the problems above into standard Gaussian matrix model correlators in the $1 / N$ expansion, where the matrix model correlators do not scale with $N$.

Since giant gravitons are similar to (di)-baryons, the combinatorial structure described above can be generalized to many other CFT's (see for example [27, 28, 29, 21, 30, 31, 32, [33) and similar results should hold there even if they are not ultimately calculable.

The paper is organized as follows:

In section 2 we give a combinatorial construction of a Fock space of open-string states on the D-brane operator. In particular we detail how the structure of spherical harmonics on the D-brane is produced. We show also that some combinatorial boundary conditions appear for the string spectrum, which begins to suggest the notion of a D-brane. The techniques to show the structure are based on turning the problem into a standard Gaussian matrix model correlation function, where the number of letters in a word does not scale with $N$ in the large $N$ limit.

Next, in section 3 we calculate the leading order anomalous dimension of a giant graviton with massless excitations. We show that the anomalous dimension is suppressed in the large $N$ limit, even in the limit of finite $g_{Y M}$.

We then consider in 0 the problem of organizing the $1 / N$ expansion for all of these states. We find a heuristic argument that suggests that there is a well defined $1 / N$ expansion. The argument is not complete and requires checking the detailed structure of the states at higher loop orders.

We end the paper with some conclusions and an appendix on the combinatoric identities that are used throughout the paper and make the problem tractable.

\section{Fock space of fluctuations on a single giant operator}

A standard picture of a D-brane is as an object in a string background where strings can end. The D-brane is a non-perturbative object, but there is a well defined perturbation theory around the object itself. Perturbatively in string theory, in the presence of a Dbrane, the Hilbert space of states is a Fock space of closed string plus open string states. This structure gets corrected when interactions are turned on: for example, open and closed string states mix, there can be a (non-perturbative) stringy exclusion principle, etc.

In order to make these statements one has to consider the fact that they are only an approximation in the geometric limit (this includes stringy geometry limits). Thus all of these statements should be corrected by the genus expansion, which in the $A d S_{5} \times S^{5}$ geometry, is the $1 / N$ expansion in the dual field theory.

Of the spectrum of strings on a D-brane, some are massless modes on a D-brane (this statement makes sense in the geometric limit, where the mass scale of these states is much smaller than the string scale). The effective action for these modes is captured by the Dirac 
Born Infeld action. A calculation of the spectrum of these modes for the giant gravitons we will study has been performed in [20].

It turns out that the spectrum of fluctuations (in the geometric limit) is independent of the size of the giant graviton, and some of these states saturate a BPS bound for some of the R-charges. Since the D3-branes do not couple directly to the dilaton, varying the coupling should not affect it's calculation, so it is conceivable that so long as gravity is weakly coupled with respect to the ADS scale ( $N$ is large), the calculation should be reliable. This suggests that these states do not change their spectrum when we take the zero coupling limit, so it should be possible to describe them simply in the free field theory (large $N$ ) limit.

These states, if we understand them in the dual theory, give rise to the structure of spherical harmonics on the giant graviton. Given this knowledge, it is possible to begin building the full set of string states ending on the D-brane.

We will now check that one can write a Fock space of excitations of a giant graviton operator which is consistent (on group theoretical grounds) with the Fock space of fluctuations of a membrane with the shape of $S^{3}$.

To begin, the BPS spherical membrane is conjectured to be given by sub-determinant operators

$$
\mathcal{O}_{D 3}^{N-k}=\epsilon^{\mu_{1}, \ldots \mu_{N},} \epsilon_{\rho_{1}, \ldots \rho_{N}} \phi_{\mu_{1}}^{\rho_{1}} \ldots \phi_{\mu_{N-k}}^{\rho_{N-k}} \delta_{\mu_{N-k+1}}^{\rho_{N-k+1}} \ldots \delta_{\mu_{N}}^{\rho_{N}}
$$

We will use the following abbreviated notation

$$
\mathcal{O}_{D_{3}}^{N-k}=\epsilon \epsilon(\phi, \ldots, \phi, \overbrace{1, \ldots, 1}^{k})
$$

to denote the operator. The $N-k$ subscript counts the number of indices saturated by the field $\phi$. In general we will treat the $\epsilon \epsilon$ convention as a multilinear operator with $N$ slots, where we can insert various fields. The purpose of this notation is mainly to keep track of how the indices are contracted without writing the indices explicitly. When the need arises we will restore the indices. This is also explained in the appendix.

The normalization of the operator $\mathcal{O}^{N-k}$ can be calculated with the combinatorial identities in the appendix to give

$$
\begin{aligned}
<O(x) \dagger O(y)>|x-y|^{2(N-k)}= & (N-k) !\left[\epsilon^{\mu_{1}, \ldots, \mu_{N-k}, \rho_{1}, \ldots, \rho_{k}} \epsilon_{\mu_{1}, \ldots, \mu_{N-k}, \sigma_{1}, \ldots, \sigma_{k}}\right. \\
& \left.\epsilon^{\nu_{1}, \ldots, \nu_{N-k}, \sigma_{1}, \ldots, \sigma_{k}} \epsilon_{\left.\nu_{1}, \ldots, \nu_{N-k}, \rho_{1}, \ldots \rho_{k}\right]}\right] \\
= & (N-k) !^{3} \delta_{\left[\sigma_{1}, \ldots, \sigma_{k}\right]}^{\left[\rho_{1}, \ldots, \rho_{k}\right]} \delta_{\left[\rho_{1}, \ldots, \rho_{k}\right]}^{\left[\sigma_{1}, \ldots, \sigma_{k}\right]} \\
= & (N-k) !^{3} k ! \delta_{\left[\rho_{1}, \ldots, \rho_{k}\right]}^{\left[\rho_{1}, \ldots, \rho_{k}\right]} \\
= & (N-k) !^{3} k !\left(\begin{array}{c}
N \\
k
\end{array}\right) k ! \\
= & (N-k) ![(N-k) ! k ! N !]
\end{aligned}
$$

We will also use the following simplifying notation

$$
\epsilon^{\mu_{1}, \ldots, \mu_{N-k}, \rho_{1}, \ldots, \rho_{k}} \epsilon_{\mu_{1}, \ldots, \mu_{N-k}, \sigma_{1}, \ldots, \sigma_{k}} \equiv \epsilon \epsilon_{k, k}() \equiv(N-k) ! \delta_{k, k}()
$$


where $k$ indicates the number of indices that are left uncontracted on each epsilon symbol, and $\delta()$ is completely antisymmetric in it's upper and lower indices ${ }^{2}$.

The contractions above can then be rewritten as

$$
\epsilon \epsilon_{k ; k} \epsilon \epsilon_{k ; k}=(N-k) ! N ! k !=(N-k) !^{2} \delta_{k ; k} \delta_{k ; k}
$$

in the notation above, contraction of indices between objects is performed by using the same letter $k$. If we want to keep the indices separate then we will use a notation where we consider changing the subscripts to $\bar{k}$, etc.

The expression $\delta_{k ; k} \delta_{k ; k}$ has a very nice $1 / N$ expansion if $k$ is fixed and does not scale with $N$, namely

$$
\begin{aligned}
\delta_{k ; k} \delta_{k ; k}= & k ! N^{k}(1-1 / N)(1-2 / N) \ldots(1-(k-1) / N) \\
= & k ! N^{k}\left[1-\left(\begin{array}{c}
k \\
2
\end{array}\right) N^{-1}+\left(\left(\begin{array}{l}
k \\
3
\end{array}\right)+\left(\begin{array}{c}
k \\
2,2
\end{array}\right)\right) N^{-2}\right. \\
& \left.-\left(\left(\begin{array}{c}
k \\
4
\end{array}\right)+\left(\begin{array}{c}
k \\
3,2
\end{array}\right)+\left(\begin{array}{c}
k \\
2,2,2
\end{array}\right)\right) N^{-3}+\ldots\right]
\end{aligned}
$$

in the expression above we use the following notation for the multinomial coefficients

$$
\left(\begin{array}{c}
l \\
n_{1}, \ldots, n_{s}
\end{array}\right)=\frac{l !}{n_{1} ! n_{2} ! \ldots n_{s} !\left(l-\sum n_{i}\right) !}
$$

and the power of $N^{-1}$ accompanying $\left(\begin{array}{c}k \\ n_{1}, \ldots n_{s}\end{array}\right)$ in the expansion is $\sum_{i}\left(n_{i}-1\right)$. Keeping $k$ finite and letting $N$ go to infinity puts us very near the maximal giant operator. Notice that the (finite) series is dominated by the first term so long as $k^{2}<N$, so it is possible to use this expansion even in this limit. This scaling is reminiscent of the dimension of operators in the Penrose limit of $A d S_{5} \times S_{5}$ [11, 34, 35, 36]. For the remainder of the paper we will consider only this possibility of $k$ small, where the above $1 / N$ expansion is well behaved.

This expansion comes from noticing that

$$
\delta_{\left[\mu_{1} \ldots \mu_{k}\right]}^{\left[\rho_{1} \ldots \rho_{k}\right]}=\sum_{\sigma}(-1)^{|\sigma|} \delta_{\mu_{\sigma(1)}}^{\rho_{1}} \ldots \delta_{\mu_{\sigma(k)}}^{\rho_{k}}
$$

is a sum of Kronecker $\delta$ over the permutations of the set $1, \ldots, k$. Each term establishes a different identification between the upper and lower indices of the $\delta_{k ; k}$. When we contract two of these tensors we have the composition of two of these permutations, with all indices contracted, which is again a permutation. Each such permutation identifies various indices among themselves. The factor of $N$ appearing in the expansion is $N^{\#(\sigma)}$, the number of cycles in the permutation, as the indices associated to each cycle are identified. The highest one has all cycles of order one. The next order has one cycle of order 2 and all others of order 1. At the third level we can get either two cycles of order 2 or one cycle of order 3 ,

\footnotetext{
${ }^{2}$ Hopefully it will be clear which convention is being used if one compares this notation with the Kronecker $\delta_{\nu}^{\mu} \sim \delta_{1,1}$ which has just one upper and one lower index.
} 
etc. The factor of $k$ ! comes about because we sum over two sets of permutations, but only the total composition of the permutations matters.

This identity can be generalized to having contractions with arbitrary matrices in the following form:

$$
\delta_{k ; k} \delta_{\tilde{k}, \tilde{k}}\left(M_{1}, \ldots, M_{k} ; \tilde{M}_{1}, \ldots \tilde{M}_{k}\right)
$$

where the $k$ upper indices and $\tilde{k}$ lower indices are contracted with the $M$, and the $k$ lower indices and $\tilde{k}$ upper indices are contracted with the $\tilde{M}$. This is equal to an alternating sum of all possible combination of traces

$$
\sum_{\sigma} \prod_{i} \operatorname{tr}\left(M_{i} \bar{M}_{\sigma(i)}\right)-\sum_{u, t \sigma} \operatorname{tr}\left(M_{t} \bar{M}_{\sigma(t)} M_{u} \bar{M}_{\sigma(u)}\right) \prod_{i \neq t, u} \operatorname{tr}\left(M_{i} \bar{M}_{\sigma(i)}\right)+\ldots
$$

where in each trace we have an equal number of distinct alternating $M, \bar{M}$ in all possible combinations (modulo cyclicity of the trace). This is described in detail in the appendix.

The sign is determined by the number of pairs of matrices $M_{i} \bar{M}_{j}$ which are fused into larger traces ( $k$ minus the total number of traces). If all the $M, \bar{M}$ are set to the identity, then we get the same sum as described above. The factor of $N^{k} N^{-s}$ comes from having exactly $k-s$ traces of the identity. The factor of $k$ ! is then the number of ways to pair a set of $k$ upper indices with $k$ lower indices. This is the number of times that $\operatorname{tr}(1)^{k}$ appears in the sum.

For the remainder of the paper we will set $k$ finite. From the above expressions it is easy to see that when $k$ is finite the total number of traces to consider is finite. If for each trace we associate a factor of $N$, the terms with the most traces dominate, so long as the number of terms with fewer traces is suppressed with respect to $N$. This again produces the estimate $k^{2} \leq N$.

Now, we are ready to start writing operators which correspond to massless excitations on the D3-brane worldvolume. For this, let us consider the following operators

$$
\mathcal{O}_{a_{n}^{\dagger} D 3}^{N-k}=\epsilon \epsilon(\phi, \ldots, \phi, \overbrace{1, \ldots, 1}^{k-1}, Z^{n})
$$

We will claim that this is an operator which corresponds to a D3-brane with one quantum of momentum $n$ on the worldsheet of the D3-brane. Since the field $\phi$ is invariant under an $S O$ (4) subgroup of the $S O(6)$ R-symmetry, we can think of this state as a highest weight state with respect to the $S 0(4) \sim S U(2) \times S U(2)$ algebra (which is of spin $(n / 2, n / 2)$ with respect to $S U(2) \times S U(2))$. Already this counting shows that there is exactly one state for each spherical harmonic of a scalar field in $S^{3}$ (the spherical harmonics come from traceless tensor products of the $(1 / 2,1 / 2)$ representation).

Since this state is a chiral operator in the free field theory, it is possible for it to be protected. Of the transverse fluctuations of the D3-brane there are four polarizations along $A d S$, and two polarizations along the $S^{5}$. The states above have no polarization along the AdS directions because they are not charged under the $S O(4)$ rotation symmetry. Thus they should correspond to transverse fluctuations along the $S^{5}$. These combine to form a complex scalar field, and the states above correspond to one of the polarizations of this 
scalar field. For each momentum there will be two polarizations, but only one is BPS, the other one is anti-BPS. Since the above states are chiral, they should be matched with the BPS polarization.

Now, we need to remember that the D3-brane is a half-BPS state, so it should be possible to act on this state with an excitation with the supersymmetries that the D3brane left unbroken. These massless states on the D-brane are acted on by the unbroken supersymmetries and generate the full multiplet of a massless field on the $S^{3}$. The D3-brane is killed by all of the conformal supersymmetries, and half of the standard supersymmetries of the superconformal algebra. The conformal supersymmetries kill any superconformal primary field, so we only need to worry about the standard supersymmetries. There are 16 in total. Half of those are broken by the D-brane, which leaves us with 8 unbroken supersymmetries. Of these, half are unbroken if the state is BPS, so it gives rise to $2^{4}$ states in a multiplet modulo descendants. It is found that there are $2^{4}$ conformal primaries in each such superprimary operator, without taking into account the supersymmetries broken by the D-brane istself. Half of these are fermionic, and half bosonic, and it is the particle content of $\mathcal{N}=4 \mathrm{SYM}$. This is enough to produce the full set of polarizations for the supersymmetric multiplet on the D3-brane, so counting the BPS states plus their descendants with respect to the unbroken supersymmetries generates the full spectrum of polarizations of the fluctuations of the D-brane and can be calculated from the above operator. Given this identification, we can rest assured that we have the complete counting of polarizations that we need to match the supergravity calculation.

Similarly, a state with two BPS quanta will be argued to be given by

$$
\mathcal{O}_{a_{m}^{\dagger} a_{n}^{\dagger} D 3}^{N-k}=\epsilon \epsilon(\phi, \ldots, \phi, \overbrace{1, \ldots, 1}^{k-2}, Z^{m}, Z^{n})
$$

and essentially, anytime we want to add one quantum we substitute one of the 1 matrices in the operator by the word $Z^{n_{i}}$ if we want it to have momentum $n_{i}$. Here we have specialized to states which are BPS and have maximum spin $(n / 2, n / 2)$, but it is very easy to write other states by using the $S O(4)$ group theory. The words that are substituting the $Z^{m}$ with the BPS transverse scalar polarization along the $S^{5}$ (so long as they do not contain $\phi, \bar{\phi}$, are fully symmetric and traceless in the $S 0(4)$ indices.

Now, there are a few details which we need to be careful about. First, we need to show that we have a Fock space of states (particles with the same quantum numbers are indistinguishable). For example $a_{n}^{\dagger} a_{m}^{\dagger}\left|D_{3}>\sim a_{m}^{\dagger} a_{n}^{\dagger}\right| D_{3}>$. This identity follows because the $\epsilon$ symbols are totally antisymmetric in their indices, so that the $\epsilon \epsilon$ operator is completely symmetric in it's entries. In essence, the states have the correct statistics to be interpreted as a Fock space of bosons. Similarly with fermionic operators, there is an extra - sign from reordering the operator, which is inherited from the statistics of the fermionic fields on the field theory.

Also, there are states with closed string gravitons and momentum $n$. These should be given by

$$
\epsilon \epsilon(\phi, \ldots, \phi, \overbrace{1, \ldots, 1}^{k}) \operatorname{tr}\left(Z^{n}\right)
$$


and anytime we want to add new gravitons we need to add a trace.

We can build multi-open string states with multi-closed string states. The closed string states will be added by multiplying the above operator by traces of other fields. Perturbatively this is the picture of a weakly coupled D-brane in string theory. If we ignore back-reaction, the open strings states and closed string states do not mix at zero coupling.

This is not all. Even though we have checked the symmetry of particle exchange on the D-brane, we still need to show that the states with one quanta are linearly independent (and orthogonal) from states with two quanta, etc, and also orthogonal with states that have gravitons in them. This is how the perturbative string states will appear if the Dbrane is weakly coupled to the background. We can only expect this statement to be true in the semi-classical limit, so it should be only true at leading order in $1 / N$. The $1 / N$ corrections will give rise to mixing between these states, already at the free field theory level $^{3}$. We need to check this fact. Also, we need to show that these states saturate all possibilities, namely that there are no more states close to the configuration with the same quantum numbers that can appear on the field theory side.

These two facts together would provide the statement that the mapping of states between the gravitational dual and the SYM theory is a unitary transformation at leading order.

For example, we could consider the operator

$$
\tilde{O}=\epsilon \epsilon(\phi, \ldots, \phi, \phi Z^{n}, \overbrace{1, \ldots, 1}^{k})
$$

which has the same quantum numbers as the operator $\mathcal{O}^{N-k} a_{n}^{\dagger} D_{3}$ and $\mathcal{O}_{D_{3}}^{N-k} \operatorname{tr}\left(Z^{n}\right)$. Naively all of these three operators have different gauge structures in how their indices are contracted, and one might believe that there are more states on the D3-brane that are apparent from the supergravity calculation.

However, there is a clever identity that shows that the last operator $\tilde{O}$ is a linear combination of the other two. To show this, we need to consider the tensor structure of

$$
\epsilon_{\mu_{1}, \ldots, \mu_{N-k}, \rho_{1}, \ldots, \rho_{k}} \phi_{\sigma_{1}}^{\mu_{1}} \ldots \phi_{\sigma_{N-k}}^{\mu_{N-k}}
$$

Since $\epsilon$ is completely antisymmetric in it's indices, it follows that the tensor above is completely antisymmetric in the $\sigma$ indices. Therefore, this operator is the same as

$$
\epsilon_{\mu_{1}, \ldots, \mu_{N-k}, \rho_{1}, \ldots, \rho_{k}} \phi_{\tilde{\sigma}_{1}}^{\mu_{1}} \ldots \phi_{\tilde{\sigma}_{N-k}}^{\mu_{N-k}} \frac{1}{(N-k) !} \delta_{[\sigma]}^{[\tilde{\sigma}]}
$$

where $[\sigma]$ is a multi-index. The factor of $(N-k)$ ! is put in to get the norm correctly. Now we remember that $\delta_{[\sigma]}^{[\tilde{\sigma}]}$ can be written in terms of $\epsilon$ symbols, so that we get an expression which is proportional to

$$
\epsilon \epsilon(\phi, \ldots, \phi)_{k ; k} \epsilon \epsilon\left(1, \ldots, 1, Z^{n}\right)_{k ; k}
$$

\footnotetext{
${ }^{3}$ The Planck constant is $1 / N$ in the dimensional reduction to five dimensions, regardless of the value of the dilaton. Thus the $1 / N$ mixing represents gravitations corrections to the D-brane
} 
The four $\epsilon$ symbols have $N-k$ of their indices contracted with the matrices $\phi, Z^{n}, 1$, and the other $k$ indices are contracted among the $\epsilon$ symbols directly. Now, in the second pair of $\epsilon \epsilon$ tensors we have $N-k-1$ indices contracted with the identity, so we get that

$$
\epsilon \epsilon\left(1, \ldots, 1, Z^{n}\right)_{k ; k} \sim \delta_{k+1 ; k+1}\left(Z^{n}\right)
$$

with one of each of the upper and lower indices contracted with $Z^{n}$. Now, if we use the permutation representation for $\delta_{k+1, k+1}$ we get terms where the upper tensor index of $Z^{n}$ is contracted with it's lower tensor, and some others where both tensor indices end contracted with free indices. The first type of term gives us $\operatorname{tr}\left(Z^{n}\right) \delta_{k ; k}$, so when we contract all of the remaining indices with the other tensor we get an operator of the form

$$
\epsilon \epsilon(\phi, \ldots, \phi, \overbrace{1, \ldots, 1}^{k})
$$

while the second type of term, once the indices are contracted gives us an operator of the form

$$
\epsilon \epsilon(\phi, \ldots, \phi, \overbrace{1, \ldots, 1}^{k-1}, Z^{n})
$$

This means that the operator we were looking at is indeed a linear combination of the other operators we were considering. This argument can be generalized for other states with open strings.

Now that we have shown that there are non-trivial identifications between states we need to check the linear independence and orthogonality of states that we described to ensure that the two spectra of states match, between supergravity with a D-brane and the dual field theory calculation.

To check this statement we need to calculate the norm of each of the states above in the large $N$ limit, where $m, n$ are fixed and finite or at most of order $\sqrt{N}^{4}$

Now, we will show the approximate orthogonality of states we have described in the large $N$ limit. The strategy we will use to prove this statement is done in the following steps: first we contract the $\phi$ and their dual field $\bar{\phi}$, giving us a contraction of four $\epsilon$ symbols. We will leave the contractions of the fields $Z$ implicit in the following way: we will write the contractions as a vev on a Gaussian matrix model. Then we will use the identity 2.16 to write the norm of the state as a vev of a gauge invariant operator in a Gaussian matrix model. We will then estimate this vev in the large $N$ limit, and from the estimate the approximate orthogonality of states will follow.

Consider for example

$$
\mathcal{O}_{a_{m}^{\dagger} a_{n}^{\dagger} D 3}^{N-k}=\epsilon \epsilon(\phi, \ldots, \phi, \overbrace{1, \ldots, 1}^{k-2}, Z^{m}, Z^{n})
$$

\footnotetext{
${ }^{4}$ This is the limit where planarity starts to fail, and it is also the limit required for understanding the plane wave geometry at finite string coupling 34, 35, 36.
} 
To calculate the norm we will do the following two point function

$$
\begin{array}{r}
\mathcal{O}_{a_{m}^{\dagger} a_{n}^{\dagger} D 3}^{N-k}(x) \mathcal{O}_{a_{m}^{\dagger} a_{n}^{\dagger} D 3}^{\dagger, N-k}(y)|x-y|^{N-k+n+m} \\
=(N-k) !^{3} \delta_{k ; k} \delta_{\bar{k}, \bar{k}}(\overbrace{1, \ldots, 1}^{k-2}, Z^{m}, Z^{n} ; \overbrace{1, \ldots, 1}^{k-2}, \bar{Z}^{m}, \bar{Z}^{n})_{Z \text { contracted }} \\
=<\mathcal{O}_{a_{m}^{\dagger} a_{n}^{\dagger} D 3}^{N-k} \mid \mathcal{O}_{a_{m}^{\dagger} a_{n}^{\dagger} D 3}^{\dagger, N-k}>
\end{array}
$$

The $Z$ can be contracted by thinking of them as matrices in a Gaussian matrix model with measure $\int[d Z][d \bar{Z}] \exp -\operatorname{tr}(Z \bar{Z})$. Now let us use the expansion 2.16 at the terms with maximum number of traces ${ }^{5}$, so that

$$
\begin{aligned}
\delta_{k ; k} \delta_{\bar{k}, \bar{k}}(\overbrace{1, \ldots, 1}^{k-2}, Z^{m}, Z^{n} ; \overbrace{1, \ldots, 1}^{k-2}, \bar{Z}^{m}, \bar{Z}^{n})= \\
(k-2)(k-3)(k-2) ! \operatorname{tr}(1)^{k-4} \operatorname{tr}\left(Z^{m}\right) \operatorname{tr}\left(Z^{n}\right) \operatorname{tr}\left(\bar{Z}^{m}\right) \operatorname{tr}\left(\bar{Z}^{n}\right) \\
+(k-2)(k-2) ! \operatorname{tr}(1)^{k-3} \operatorname{tr}\left(Z^{m} \bar{Z}^{m}\right) \operatorname{tr}\left(Z^{n}\right) \operatorname{tr}\left(\bar{Z}^{n}\right) \\
+(k-2)(k-2) ! \operatorname{tr}(1)^{k-3} \operatorname{tr}\left(Z^{n} \bar{Z}^{m}\right) \operatorname{tr}\left(Z^{m}\right) \operatorname{tr}\left(\bar{Z}^{n}\right) \\
+(k-2)(k-2) ! \operatorname{tr}(1)^{k-3} \operatorname{tr}\left(Z^{m} \bar{Z}^{n}\right) \operatorname{tr}\left(Z^{n}\right) \operatorname{tr}\left(\bar{Z}^{m}\right) \\
+(k-2)(k-2) ! \operatorname{tr}(1)^{k-3} \operatorname{tr}\left(Z^{n} \bar{Z}^{n}\right) \operatorname{tr}\left(Z^{m}\right) \operatorname{tr}\left(\bar{Z}^{m}\right) \\
+(k-2) ! \operatorname{tr}(1)^{k-2} \operatorname{tr}\left(Z^{n} \bar{Z}^{n}\right) \operatorname{tr}\left(Z^{m} \bar{Z}^{m}\right)+(k-2) ! \operatorname{tr}(1)^{k-2} \operatorname{tr}\left(Z^{n} \bar{Z}^{m}\right) \operatorname{tr}\left(Z^{m} \bar{Z}^{n}\right)
\end{aligned}
$$

In the above we have counted the multiplicity for each term that appears in the sum. Now we can evaluate these terms in the large $N$ limit. To do this, it is convenient to rescale $Z=\sqrt{N} z$, and extract a factor of $N$ from each trace. We are left with

$$
\begin{aligned}
\delta_{k ; k} \delta_{\bar{k}, \bar{k}}(\overbrace{1, \ldots, 1}^{k-2}, Z^{m}, Z^{n} ; \overbrace{1, \ldots, 1}^{k-2}, \bar{Z}^{m}, \bar{Z}^{n}) & = \\
N^{k+n+m}(k-2) ! & <\frac{1}{N} \operatorname{tr}\left(z^{m} \bar{z}^{m}\right) \frac{1}{N} \operatorname{tr}\left(z^{n} \bar{z}^{n}\right)>+\ldots
\end{aligned}
$$

now, in the Gaussian matrix model in the large $N$ limit we can use factorization of correlation functions to estimate the above correlator. It turns out that the only contribution is exactly from the term left in the line above (we assume $n \neq m$ ), which is of order 1 ( a function of $n, m$ alone, with a power series in $N^{-2}$ ). Once the leading power of $N$ is extracted, the terms encompassed by the ... symbol have zero vev in the strict large $N$ limit (fewer traces have suppressions of $1 / N$ ), so they are subleading with respect to the first term. The $N$ counting of the subleading terms is straightforward: each trace counts as a disconnected disk. Contractions between different traces bring factors of $1 / N^{2}$ from handles joining the disconnected disks.

The terms with fewer traces also contribute to the norm. For each trace we get a factor of $N$, so terms with fewer traces will have a $1 / N$ suppression. From the above considerations, the subleading contribution to the norm will come from the correlator with

\footnotetext{
${ }^{5}$ This is the place were keeping $k$ finite in the large $N$ limit simplifies the argument
} 
the following trace structure $\operatorname{tr}(1)^{k-2} \operatorname{tr}\left(z^{m} \bar{z}^{n} z^{n} \bar{z}^{m}\right)$. In general we will obtain a power series in $N^{-1}$ and not $N^{-2}$. This suggests already that if there is an interpretation in terms of Riemann surfaces suppressed by the genus, there are contributions of worldsheets with boundaries.

Now let us consider an overlap between the state above, and another state, also with two quanta but with different momenta $\tilde{n}, \tilde{m}$, but with the same momentum $\tilde{n}+\tilde{m}=n+m$.

We want to consider the overlap in the normalization of the states

$$
<\mathcal{O}_{a_{m}^{\dagger} a_{n}^{\dagger} D 3}^{N-k} \mid \mathcal{O}_{a_{\tilde{m}}^{\dagger} a_{\tilde{n}}^{\dagger} D 3}^{\dagger, N-k}>
$$

We repeat the steps outlined above, and remember to keep the terms with maximum number of traces. We will thus get contributions of the general form

$$
N^{k+m+n}<\frac{1}{N} \operatorname{tr}\left(z^{m} \bar{z}^{\tilde{m}}\right) \frac{1}{N} \operatorname{tr}\left(z^{n} z^{\tilde{n}}\right)>\sim N^{k+m+n-2}
$$

because at leading order in the large $N$ limit the one point functions $<\frac{1}{N} \operatorname{tr}\left(z^{n} z^{\tilde{n}}\right)>$ vanish. Thus they start contributing at order $N^{-2}$.

However, when we consider terms with fewer traces, there are terms of the type

$$
\operatorname{tr}(1)^{k-2} \operatorname{tr}\left(Z^{m} \bar{Z}^{\tilde{m}} Z^{n} \bar{Z}^{\tilde{n}}\right)+(n \leftrightarrow m)
$$

which are of order $N^{k+m+n-1}$. It takes a while to see which contractions contribute, but it is found that the correlator does receive contributions from planar diagrams.

Thus the matrix of overlaps for the states above is roughly of order

$$
<O \mid O^{\dagger}>\sim N^{k+m+n}\left(\begin{array}{cc}
O(1) & O(1 / N) \\
O(1 / N) & O(1)
\end{array}\right)
$$

This shows that the two states are approximately orthogonal. Notice that for these operators, the corrections to the norm and mixing both give an expansion in $1 / N$ and not $1 / N^{2}$, consistent with an open string interpretation.

In general it can be shown that the norm of an operator with total momentum $n=$ $n_{1}+\cdots+n_{s}$ is of order $(N-k) !^{3} N^{k+n}$ times factors of order one. If one considers another operator with the same momentum, the norm will also be of roughly the same size. To consider the overlap we will find that in the maximum number of traces at least one of the traces will contain different powers of $z, \bar{z}$. Thus the matrix model factorized amplitude vanishes, and only the subleading terms contribute. These can have less traces, and like the case above we can obtain overlap terms which are of order $1 / N$ with respect to the original norm of the states.

Now, let us consider comparing a state of a D-brane plus a closed string state with one of a D-brane with an open string state. For example, $a_{n}^{\dagger} \mid D_{3}>$ and $\mid D 3>\times O_{\text {Closed }}$. These operators are respectively $\epsilon \epsilon\left(\phi, \ldots, \phi, 1, \ldots, 1, Z^{n}\right)$ and $\epsilon \epsilon\left((\phi, \ldots, \phi, 1, \ldots, 1,1) \operatorname{tr}\left(Z^{n}\right)\right.$. The norm squared of the first state is of order $(N-k) !^{3} N^{n+k}$, which is of the same order of magnitude as the second. The overlap amplitude reduces to the matrix model computation of

$$
\delta_{k, k} \delta_{\tilde{k}, \tilde{k}}\left(1, \ldots 1, Z^{n} ; 1, \ldots 1\right) \operatorname{tr}\left(\bar{Z}^{n}\right) \sim \operatorname{tr}(1)^{k-1} N^{2} \operatorname{tr}\left(z^{n}\right) \operatorname{tr}\left(\bar{z}^{n}\right) \sim N^{k-1}
$$


From here, we see that the overlap of D-branes with an open string state and a D-brane with a closed string state are also suppressed by $1 / N$, and hence at leading order the states are orthogonal, but the mixing is again of order $1 / N$ and not $1 / N^{2}$

In general, for closed string states the contribution from factorized amplitudes with the maximum number of traces in the matrix model vanishes, due solely to the presence of the closed strings. The correction for a state with $s$ gravitons is a factor of $N^{-2 s}$, but we also have $2 s$ more traces to consider (the traces of the graviton states and their's complex conjugates), so the $N$ counting for the norm is still $N^{k+n}$ where $n$ is the total momentum.

Now, let us consider a state of the form

$$
\epsilon \epsilon(\phi, \ldots, \phi, \overbrace{1, \ldots, 1}^{k}, Z^{m} \phi Z^{n})
$$

with one $\phi$ sandwiched between two words of the type $Z^{k}$. We will ask whether this state is approximately orthogonal to a state

$$
\epsilon \epsilon(\phi, \ldots, \phi, \phi, \overbrace{1, \ldots, 1}^{k-1}, Z^{m+n})
$$

or to a state

$$
\epsilon \epsilon(\phi, \ldots, \phi, \phi, \overbrace{1, \ldots, 1}^{k-2}, Z^{m}, Z^{n})
$$

These are the two types of $Z$ configurations which can maximize the contributions from the "planar diagrams" associated to $Z$.

If it is, we can interpret the defect $\phi$ sandwiched between the $Z$ as starting to build the open string oscillators of the string with polarization along $\phi$, and momentum $n+m^{6}$.

The first thing we need to take care of is the norm of the state. We need to worry whether the norm comes primarily from contracting the two $\phi, \bar{\phi}$ which are special, or from "mixing" with the other $\phi$. It can be easily shown that the contribution without mixing (with maximum umber of traces) reduces to the Gaussian matrix model vev

$$
(N-k) !^{3} N^{k+m+n+1}<1 / N \operatorname{tr}\left(z^{n} x z^{m} \bar{z}^{m} \bar{x} \bar{z}^{n}\right)>
$$

with two matrices. This is essentially the same type of expression for this states as 2.33 . Here the special $\phi$ has been replaced by the matrix model variable $x$.

The mixing term comes as

$$
(N-k-1) !^{3}(N-k)^{2} N^{m+n+1} \delta_{k+1, k+1} \delta_{k+1, k+1}\left(1, \ldots 1, \phi, Z^{n} \phi Z^{m} ; 1, \ldots 1, \bar{\phi}, \bar{Z}^{m} \bar{\phi} Z^{m}\right)
$$

which gives us a set of two terms with maximum number of traces

$$
\begin{array}{r}
(N-k) !^{3} N^{-1} N^{k-1}\left[\operatorname{tr}\left(\phi \bar{Z}^{m} \bar{\phi} \bar{Z}^{n}\right) \operatorname{tr}\left(\bar{\phi} Z^{n} \phi Z^{m}\right)\right. \\
\left.+: \operatorname{tr}(\phi \bar{\phi}): \operatorname{tr}\left(\bar{Z}^{n} \phi Z^{m} \bar{Z}^{m} \bar{\phi} \bar{Z}^{n}\right)\right]
\end{array}
$$

\footnotetext{
${ }^{6}$ In the plane wave limit this should become precise when we consider the wave functions of the defect inside the string of $Z$ 's
} 
The normal ordered symbol above is to remind us to contract the special $\phi$ with the $\bar{\phi}$ which are contracted with the $\epsilon \epsilon$ tensors. The first term is of order at most $N^{n+m+2}$ if we use planar diagrams, and this is suppressed at $1 / N$ with respect to the calculation above. These planar terms actually come from contracting the two exceptional $\phi$ 's together, so part of them are just subleading because of trace structure. The terms with the mixed contractions are suppressed at least by $N^{-2}$ with respect to these, which gives a total suppression of order $N^{-3}$. The second term we need to keep the terms with mixed contractions and they give $(N-k) !^{3} N^{k-2} N^{n+m+2-2}$, so these are suppressed by $N^{-2}$.

Orthogonality with respect to the other operators reduces to evaluating the following

$$
\delta_{k, k} \delta_{k, k}\left(1, \ldots, 1, Z^{n} \phi Z^{m} ; \bar{Z}^{n}, \bar{Z}^{m}, \bar{\phi}, 1, \ldots 1\right)
$$

and

$$
\delta_{k, k} \delta_{k, k}\left(1, \ldots, 1, Z^{n} \phi Z^{m} ; \bar{Z}^{n+m}, \bar{\phi}, 1, \ldots 1\right)
$$

and it is easy to see that these are suppressed contributions, either because we have less traces, or there are non-planar diagrams to evaluate. The rest of the calculation is pretty straightforward and orthogonality follows.

It is easy to extend this argument to the presence of more $\phi$ which are sandwiched between the $Z$, and also to show that configurations with $\phi$ in different positions in the word are also orthogonal to each other. In the end, we build string states by replacing one of the 1 symbols with a word, with the additional condition that it can not begin or end with the letter $\phi$ which builds the giant graviton. Because we turn the problem in the end to a Gaussian correlation function in the large $N$ limit, different orders of the letters in the word matter, and different words produce different states.

To consider fluctuations orthogonal to the brane along the $A d S$ directions, we can attach a covariant derivative $\left(Z^{m}\left(D_{\mu} Z\right) Z^{n}\right)$. More covariant derivatives in the same word results in large planar anomalous dimensions, so these build oscillators and not "spherical harmonics" along the $A d S$ space. This is what shows that the D-brane is localized in the AdS directions. The zero mode $D_{\mu}$ is special. We have to contract it with one of the $\phi$, but this just generates the descendants of the conformal primary. We can think of this property as giving the correct boundary conditions for the oscillators that are transverse to the brane along the $A d S$ directions, showing that we have only one massless mode along $A d S$ and not infinitely many. Notice that in some sense the boundary condition for $\phi$ is implemented by the identities we considered, where a $\phi$ at the end of the open string should not be considered as part of the open string itself.

The planar diagrams associated to the words of each string will produce the same type of perturbation series for anomalous dimensions than closed strings do. In this sense, if traces generate a full string spectrum with all possible polarizations, it should be the case that the planar anomalous dimension for words do the same thing.

This concludes our proof that the combinatorics of the spectrum of states of large subdeterminants has the right properties to be describing a single giant graviton which is localized in $A d S$ space and that the open string states have enough polarizations to generate the full tower of oscillators. 
We need to supplement this with having the geometric oscillations on the D-brane to be approximately BPS. This can not be checked in free field theory nor using just planar diagrams. Also, the statements about large anomalous dimensions for insertions of various covariant derivatives, or different word orderings in a single slot are heuristic. A full check is beyond the scope of this paper.

The assumption we have made above is that if we can neglect the fields $\phi$ which make the giant graviton to calculate an anomalous dimension (except perhaps for boundary conditions), then we have the requisite number of sates to generate a full open string spectrum on the D-brane. We will now show that the one loop anomalous dimension of the operators 2.17 is protected. This shows that the giant graviton operator has the correct shape, and it will provide evidence that Feynman diagrams involving the giant graviton $\phi$ are suppressed in the large $N$ limit and lead to a well defined $1 / N$ expansion.

\section{One loop anomalous dimensions}

When considering the large $N$ limit of a theory, it is important to ask whether perturbation theory for a particular object is useful at all. Witten has argued that in generic large $N$ theories baryonic states will have a mass of order $N[26]$, which suggests that in general the standard perturbation theory will not be well behaved, because diagrammatic corrections will grow with powers of $N$.

In the case we want to analyze there is room for improvement on this general statement. The main reason is that the state we start with is BPS, which in perturbation theory should imply that even though the individual contributions of a given diagram grow with $N$, the contributions to the mass of a state cancel when all Feynman graphs that contribute are added together.

When we consider the operators like 2.17 and 2.18 the leading contributions (of order $N$ ) to the anomalous dimensions will cancel because of supersymmetry, and then the question is how suppressed are the diagrams that contribute at the subleading order to the dimension of the operator.

If they are sufficiently suppressed there are two possibilities: the result exactly cancels, but it might require small mixing with other states and there is a $1 / 4 \mathrm{BPS}$ operator which is near the one that we started, or the perturbative expansion is valid even in the strong 't Hooft coupling limit, and the expansion is suppressed with respect to the natural 't Hooft

coupling. It is very plausible that some of these states are BPS, as multi trace $1 / 4 \mathrm{BPS}$ operators which are protected have been found in [37, 38].

Now, we turn to the calculation of the anomalous dimension itself. The first thing we notice is that the operator as described is composed only of chiral fields, so there is a chance that the operator is non-vanishing in the chiral ring. For these chiral operators the D-term and vector exchange contributions cancel each other 39], and only the F-terms are necessary to calculate the anomalous dimension of the operator. When we do a first order perturbation theory calculation, we need to choose one of the $\phi$ from the giant graviton operator to contract with the perturbation. Once we find the logarithmic contribution to 
the two point function, it needs to be contrasted with the norm of the state to find the anomalous dimension.

To calculate the norm, we can also choose to write the norm in the following form

$$
\left|O_{a_{n}^{\dagger} \mid D_{3}>}^{N-k}\right|^{2} \simeq \frac{(N-k) !^{3}}{(N-k)^{2}} \delta_{k+1, k+1} \delta_{k+1, k+1}\left(\phi, 1, \ldots, 1, Z^{n} ; \bar{\phi}, 1 \ldots 1, \bar{Z}^{n}\right)_{m m}
$$

where the right hand side is a Gaussian matrix model calculation. The $Z$ and $\phi$ are all contracted. The main contribution to the norm comes from the maximum number of traces, and it is given by

$$
\begin{aligned}
\left|O_{a_{n}^{\dagger} \mid D_{3}>}^{N-k}\right|^{2} \simeq & \frac{(N-k) !^{3}}{N-k}(k-1) ! N^{k-1} \operatorname{tr}(\phi \bar{\phi}) \operatorname{tr}\left(Z^{n} \bar{Z}^{n}\right) \\
& \sim \frac{(N-k) !^{3}}{(N-k)^{2}}(k-1) ! N^{k-1+(1+n)+(1+1)}
\end{aligned}
$$

and to leading order in $N$ it can be seen that his calculation agrees with 2.30 .

To calculate the one loop anomalous dimension, we can write all the $x$ dependence in a factorized form and we can reduce the problem to a matrix model computation. With the same normalization of the fields as above, the anomalous dimension will be proportional to the ratio

$$
\frac{(N-k)<\delta_{k+1, k+1} \delta_{k+1, k+1}\left(\phi, \ldots, Z^{n} ; \bar{\phi}, \ldots Z^{n}\right) g_{Y M}^{2}:[X, \phi][\bar{Z}, \bar{\phi}]:>_{m m}}{<\delta_{k+1, k+1} \delta_{k+1, k+1}\left(\phi, \ldots, Z^{n} ; \bar{\phi}, \ldots Z^{n}\right)>_{m m}}
$$

the :: notation indicates normal ordering, no self contraction of the F-term vertices. Most of the combinatorial factors cancel between the top and bottom lines, as they involve the same contractions of the fields $\phi, \bar{\phi}$ between the giant gravitons. The only non-vanishing combinatorial factor $(N-k)$ is the one associated to choosing one field $\phi$ from the giant graviton to contract with the perturbation.

The naive $N$ dependence of the interaction vertex above is of order $g_{y m}^{2} N^{3}$, or $\lambda N^{2}$. If we take into account the non-planarity of the contractions we get a $1 / N^{4}$ suppression, so the total contribution to the anomalous dimension is of order $g_{Y M}^{2} N^{4} / N^{4} \sim g_{Y M}^{2}$. We need to consider the top contribution in more detail. The trace structure of the matrix model computation is as follows

$$
\begin{array}{r}
{\left[\operatorname { t r } ( 1 ) ^ { k - 1 } \left[\operatorname{tr}\left(Z^{n} \bar{Z}^{n}\right) \operatorname{tr}(\phi \bar{\phi})+\operatorname{tr}\left(Z^{n} \bar{\phi}\right) \operatorname{tr}\left(\phi \bar{Z}^{n}\right)\right.\right.} \\
\left.\left.-\operatorname{tr}\left(Z^{n} \bar{Z}^{n} \phi \bar{\phi}\right)-\operatorname{tr}\left(Z^{n} \bar{\phi} \phi \bar{Z}^{n}\right)\right]+\ldots\right]: \operatorname{tr}([Z, \phi][\bar{Z}, \bar{\phi}]):
\end{array}
$$

the terms denoted with ... will have less powers of $\operatorname{tr}(1)$, so they will be suppressed, and can have more traces, but then the non-planar suppression is even higher.

One can check that even though the terms of the form $\operatorname{tr}\left(Z^{n} \bar{\phi}\right)$ only contributes at a subleading order to the norm of the state, when one considers the contribution to the anomalous dimension of the operator it actually cancels the contribution from the first term, because contractions with the interaction vertex come with opposite signs. Also, the terms which could give a bigger contribution to the anomalous dimension than the first 
two (because of this cancellation) are doubly non-planar suppressed as well, because in the vertex $: \operatorname{tr}([Z, \phi][\bar{Z}, \bar{\phi}]):$ the two chiral terms appear together, whereas in the trace structure of the matrix model trace they have an order where the chiral and anti-chiral fields are alternating.

Putting all of the calculation together we see that the anomalous dimension of the operator is of order at most $g^{2} N^{-1}$ to leading order. This argument can be extended to having more open strings with their momentum along $Z$. The result is that at least to leading order, perturbation theory for these states gives very small corrections, and they become smaller in the large $N$ limit. We take this as evidence that the identification of states we have done can interpolate between strong and weak 't Hooft coupling. Clearly, this matches the result from fluctuations around the giant graviton [20]

\section{Organizing the $1 / N$ expansion.}

In this section we will give a description of how to organize the $1 / N$ expansion of the giant graviton operators with their stringy fluctuations in general.

The generic operator we have been discussing can be written in the following form

$$
O^{N-k} \sim \epsilon \epsilon(\phi, \ldots, \phi, \overbrace{1, \ldots, 1}^{k-l}, w_{1}, w_{2}, \ldots, w_{l}) \operatorname{tr}\left(s_{1}\right) \ldots \operatorname{tr}\left(s_{m}\right)
$$

where the $w_{l}, s_{i}$ are words, and $w$ does not begin or end in the symbol $\phi$.

Let us consider first the case where there are no fields $\phi, \bar{\phi}$ in the words $w_{i}, s_{l}$. Then the free contraction of two of these operators (after we contract all the $\phi, \bar{\phi}$ ) will give rise to the following Gaussian matrix model amplitude

$(N-k) !^{3}<\delta_{k ; k} \delta_{\bar{k} ; \bar{k}}\left(1, \ldots, 1, w_{1}, w_{2} \ldots w_{l} ; 1, \ldots 1, \tilde{w}_{1}, \ldots, w_{a}\right) \operatorname{tr}\left(s_{1}\right) \ldots \operatorname{tr}\left(s_{m}\right) \operatorname{tr}\left(\tilde{s}_{1}\right) \ldots \operatorname{tr}\left(\tilde{s}_{b}\right)>$

This expression above is some sum of traces where each word in the expression has a finite number of letters (or of order up to $\sqrt{(} N)$ if one wants to study the plane wave limit). This expression 4.2 has a well defined $1 / N$ expansion. The individual trace combinations have a $1 / N^{2}$ expansion, but since the expression above has various trace types, these give rise to a $1 / N$ expansion.

Similarly, we can consider expressions where some of the words have fields $\phi, \bar{\phi}$ (but not at the beginning or end of the $w_{i}$ ), and it can also be the case that $k \neq \tilde{k}$, but that $k-\tilde{k}$ is finite. From here, most of the $\phi, \bar{\phi}$ will be contracted as usual, but let us assume

that there are $t_{1}, t_{2}=t_{1}+k-\tilde{k}$ of these which are contracted with the words inside the traces.

These terms will give rise to the following type of matrix model amplitudes

$$
\begin{array}{r}
\left(N-k-t_{1}\right) !^{2} C\left(k, t_{1}, t_{2}\right) \delta_{k+t_{1}, k+t_{1}} \delta\left(\tilde{k}+t_{2}, \tilde{k}+t_{2}\right) \overbrace{\hat{\hat{\phi}}, \ldots, \hat{\phi}}^{t_{1}}, 1, \ldots, 1, w_{1}, \ldots, w_{n} ; \\
\overbrace{\hat{\bar{\phi}}, \ldots, \hat{\bar{\phi}}}^{t_{2}}, 1, \ldots \tilde{w}_{1}, \ldots \tilde{w}_{a}) \operatorname{tr}\left(s_{1}\right) \ldots \operatorname{tr}\left(s_{l}\right) \operatorname{tr}\left(\tilde{s}_{1}\right) \ldots \operatorname{tr}\left(\tilde{s}_{b}\right)
\end{array}
$$


where the $\hat{\phi}$ fields are not allowed to be contracted with the $\hat{\bar{\phi}}$ fields. In this notation, $C\left(k, t_{1}, t_{2}\right)$ is a combinatorial constant that counts the number of contractions of this type.

This constant is given by

$$
C\left(k, t_{1}, t_{2}\right)=\frac{(N-\tilde{k}) !}{t_{2} !}\left(\begin{array}{c}
N-k \\
t_{1}
\end{array}\right)
$$

It can be checked explicitly (using $N-k-t-1=N-\tilde{k}-t_{2}$ ) that the above expression for $C$ is symmetric under the exchange of the two operators one can be analyzing.

Now, we want to show that as we sum over all possible values of $t_{1}$, successive terms give rise to terms of order $1 / N^{t_{1}}$ in the $1 / N$ expansion.

The total combinatorial coefficient in front of the operator is

$$
\frac{(N-\tilde{k}) !(N-k) !\left(N-k-t_{1}\right) !}{t_{2} ! t_{1} !}
$$

which when compared to the same expression with different values of $t_{1}$ is of order

$$
\left(N-k-t_{1}\right) ! /\left(N-k-\tilde{t}_{1}\right) ! \sim \frac{(N-k) ! /(N-k) \ldots\left(N-k-t_{1}+1\right)}{(N-k) ! /(n-k) \ldots\left(N-k-\tilde{t}_{1}+1\right)} \sim N^{-\left(t_{1}-\tilde{t}_{1}\right)}
$$

with the coefficient having a well defined $1 / N$ expansion.

Now, we need to understand the contractions of the $\hat{\phi}$ and the $\hat{\bar{\phi}}$. First of all, there are configurations with more traces which are possible, $k+t_{1}$ of them. This gives a factor of $N^{k+t_{1}}$ from counting traces. Also, each pair $\phi, \hat{\bar{\phi}}$ gives an extra factor of $N$, so combining all the factors of $N$ it seems as if these amplitudes are of order $N^{2 t_{1}-t_{1}}$, times some fixed power of $N$. This is the combinatorial growth of Feynman diagrams that is responsible for possible bad behavior in the $1 / N$ expansion.

However, the definition of the operators should be in normal ordered form if selfcontractions of the operator are allowed (this is automatic for chiral operators), and we have also set the rule that the $\hat{\phi}$ can not be contracted with the $\hat{\bar{\phi}}$, so that the $\hat{\phi}$ can only contract with letters in the $\tilde{w}$ and $\tilde{s}$, and similarly the $\hat{\phi}$ only contract with the $w$ and $s$. Also, $w$ is such that it can not begin or end on a $\phi$ operator, so if the $\hat{\bar{\phi}}$ appears in the same trace as a $w$ all of the possible contractions are non-planar in the matrix model. Because of these non-planarity, one gets at least an extra factor of $N^{-2 t_{1}}$ with respect to planar amplitudes, and the simplest case is to consider contractions where the words $w, \tilde{w}$ are missing.

All together, the amplitude is suppressed in $N^{-t_{1}}$. Notice also that when we include interactions, the interaction vertex is of order $N^{2} \lambda$, and can contribute to self-energy diagrams at order $\lambda$ (this is because the contraction will be non-planar with respect to the large $N$ vev of the perturbation), which have the same combinatorial structure as above, but without the additional non-planar suppression. However, supersymmetry should ensure that the pure self energy of the $\hat{\phi}, \bar{\phi} \phi$ will cancel (because these diagrams would contribute to the anomalous dimension of the protected half-BPS operator otherwise), so diagrams with self-energy contributions are also suppressed by powers of $N^{-t_{1}}$, because they can only involve dressed propagators for the same diagrams as we had above. 
Also, if we study the trace structure of operators of the type above in the presence of interactions, the interactions leave most of the fields $\phi$ untouched, and if we think of them as partons, their color structure is still completely antisymmetric in lower and upper indices for all but a finite number of the $\phi$. This means that in perturbation theory if we begin in the class of operators we have been studying, we stay in this class of operators. In this sense, perturbation theory is closed within the class of operators we have been studying.

Mixing with operators of different trace structure (combinations of giant gravitons with distinct sizes for example) are allowed. However, if they are not of the shape we have been describing, their overlap with the given operators above is suppressed much more than any polynomial behavior in $N$. For other combinations of giant gravitons it is exponentially suppressed in $N$, and mixing with these states is to be considered as nonperturbative. Thus, in perturbation theory we have described a sector which can be studied independently and has a well defined $1 / N$ expansion.

Putting all of the information together: the perturbation theory around the giant graviton operators seems to be well defined, and it is described by a sector of the large $N$ limit of the $\mathcal{N}=4$ SYM. Clearly, the argument above is sketchy as we have not discussed issues with mixed combinatorics, "overlapping divergences". To first order it is correct. It is certainly worthwhile to explore this expansion in more detail, but it is beyond the scope of the present article.

To summarize, there are two main effects contributing for the large $N$ counting: combinatorial enhancement of diagrams, together with non-planar suppressions. The planar diagrams contributing to the self-energy of the "background" D-brane itself cancels, and only diagrams that mix the D-brane and the defects on it contribute. The non-planar suppressions induced by these diagrams dominate over the combinatorial enhancement, and the D-brane with defects has a well defined $1 / N$ expansion.

Considering also the combinatorial structure of planar anomalous dimensions together with restrictions on the shape of operators, the D-brane is producing 'boundary conditions' for letters in the words $w_{i}$. These planar diagrams are of the same type as for closed string states, and thus should lead to the same type of spectrum of anomalous dimensions (strings with boundary conditions).

\section{Conclusion}

In this paper we have shown that the conjectured dual operator to a giant graviton has all the requisite properties to describe a D-brane. This is, there is a collection of operators which can be considered as stringy excitations of the D-brane ${ }^{7}$, and it has the correct spectrum of low energy (massless) excitations on it's worldvolume. This constitutes a test of shape of the D-brane. This is important as it helps us understand the nature of locality in higher dimensions from the holographic dual theory.

\footnotetext{
${ }^{7}$ The spectrum of fluctuations of the D-brane state approximates a Fock space of string states in the large $N$ limit
} 
Also, we have presented evidence that the perturbative diagrams contributing to stringy amplitudes have a well defined $1 / N$ expansion, which can be made explicit by using matrix model techniques.

Clearly these combinatorial properties will be the same for other SCFT in four dimensions, except that some of the exact details will have to change because of the different matter content of the theory.

Supersymmetry, as in other examples [11], was the key to understanding the giant graviton operators: without self energy diagram cancellations the perturbation theory does not seem to behave well at all, and would conform to a more standard picture of baryons in large $N$ theories [26].

Many puzzles still remain open.

It would be interesting if one could show that there is a spectrum of excitations which is $1 / 4 \mathrm{BPS}$ protected. This might require taking into account some amount of mixing between states before one can decide weather this is true or not, and judging from [37, 38], this will almost certainly be the case.

Here we have shown that to leading order there is such a spectrum of states. However, it does not determine the position of the D-brane fully, as giant gravitons of different sizes have the same spectrum of fluctuations independent of their size. It could be the case that interactions between the waves can tell them apart. With the methods presented here it should be possible to check the non-linear terms in the DBI action. This is, the massless open string scattering on the D-brane worldvolume.

There are other configurations of giant graviton states with less supersymmetry in the geometric limit [40], which have also been studied in [30, 31]. If these states will be protected, it is probably true that the spectrum of $1 / 4$ BPS states described above should exist. There is to my knowledge no candidate dual operators to these other giant graviton states in the literature. Considering that for these type of objects non-planar diagrams dominate, it will require a lot of combinatorial dexterity to show that any such operator can have a well defined $1 / N$ expansion. Even for a giant graviton which is less than near-maximal, the combinatorial problem is much harder than what has been presented here.

In this paper, we have mentioned the possibility of studying the plane wave limit in the presence of a giant graviton. This is a natural scenario to study as it is a limit which simplifies the diagramatics considerably: it is known that the spectrum of states can be controlled very well, and it would be interesting if one can compare amplitudes in the presence of the D-brane. These tests should finish determining the position of the brane and showing that the boundary conditions set by the D-brane operator are the correct ones. The reason this is tests the location of the D-brane is because the plane wave states are very localized in a small tube inside the AdS spacetime, so interactions with the closed string states on the plane wave are unsuppressed only if the D-brane contains the null trajectory along which one is taking the Penrose limit.

Finally, it would be very interesting if one could extend these ideas to the presence of many D-branes on top of each other. This should serve as a test on how the $U(N)$ gauge theory on the D-brane worldvolume arises. The comparison between states in the 
geometric limit and states in the gauge field theory should be such that one obtains the $U(N)$ invariant sector of a Fock space of strings in the adjoint of $U(N)$. Understading the map of states could be a quite complicated combinatorial problem. This can be made explicit if one considers a simpler case with just two D-branes on top of each other, both with maximum angular momentum and given by the operator $O_{D 3}^{2}$. Then the connected diagram contribution to the norm of the state dominates over the product of the norm of the individual states. In essence, one can not consider the state as factorized in any sense, unlike when one takes the product of two ordinary graviton states. It is possible that this behavior is responsible for producing the enhanced gauge symmetry when the two D-branes are on top of each other, but at this point, this is just speculation.

\section{Acknowledgments}

I would like to thank V. Balasubramanian, S. Cherkis, R. Gopakumar, A. Hashimoto, I. Klebanov and J. Maldacena for various useful discussions. Research supported in part by DOE grant DE-FG02-90ER40542

\section{A. Combinatorial identities}

Here we will derive some combinatorial identities which are used in the paper. These are built out of the totally antisymmetric tensor $\epsilon_{\mu_{1}, \ldots, \mu_{N}}$ in $N$ entries, which is a gauge invariant tensor for $S U(N)$.

The components of $\epsilon$ are

$$
\epsilon_{\mu_{1}, \ldots, \mu_{N}}=\left\{\begin{array}{c} 
\pm 1, \text { If all of the } \mu_{i} \text { are different } \\
\text { 0Otherwise }
\end{array}\right.
$$

The sign of the symbol is determined as follows

$$
\epsilon_{\sigma(1), \ldots, \sigma(N)}=(-1)^{|\sigma|}
$$

for $\sigma$ a permutation of $1, \ldots, N$, and $|\sigma|$ is the parity of the permutation.

We define a similar object with raised indices. We can similarly define an $\epsilon$ tensor with all indices raised.

Many of the calculations in the paper involve the contraction of $\epsilon$ tensors. First, consider the contraction of $N-k$ indices of two $\epsilon$ tensors

$$
\epsilon^{\mu_{1}, \ldots, \mu_{N-k}, \rho_{1}, \ldots, \rho_{k}} \epsilon_{\mu_{1} \ldots, \mu_{N-k}, \nu_{1}, \ldots, \nu_{k}}=\epsilon \epsilon_{\nu_{1}, \ldots, \nu_{k}}^{\rho_{1}, \ldots, \rho_{k}}
$$

The contraction above defined the $\epsilon \epsilon$ symbol. Now let us evaluate the components of $\epsilon \epsilon$. We notice immediately that the tensor is completely antisymmetric in both it's lower and upper indices. So if any of the upper or lower indices is repeated the result is zero.

Secondly, once we have fixed $\rho_{1}, \ldots, \rho_{k}$ all different, then for the terms to contribute in the sum we have to sum over all possible combinations of $\mu_{i}$ which are different from 
the $\rho_{j}$. It also follows that the result is zero unless the set of the $\nu_{j}$ is the same set as the $\rho_{i}$, with permutations allowed.

Given such a permutation $\sigma\left(\rho_{j}\right)$, we get from A.2 that when we evaluate the tensor we find

$$
\epsilon \epsilon_{\sigma\left(\rho_{1}\right), \ldots, \sigma\left(\rho_{k}\right)}^{\rho_{1}, \ldots, \rho_{k}}=(N-k) !(-1)^{|\sigma|}
$$

The $(N-k)$ ! is the number of possible different combinations of $\mu_{1}, \ldots \mu_{N-k}$ that can contribute. They all contribute with the same sign. Since the tensor is different from zero only when the upper and lower indices coincide, and the components are a fixed number (up to a sign), we define the standard symbol

$$
\epsilon \epsilon_{\nu_{1}, \ldots, \nu_{k}}^{\rho_{1}, \ldots, \rho_{k}}=(N-k) ! \delta_{\left[\rho_{1}, \ldots, \rho_{k}\right]}^{\left[\mu_{1}, \ldots, \mu_{k}\right]}
$$

and it can also be shown by comparing the components of tensors that

$$
\delta_{\left[\rho_{1}, \ldots, \rho_{k}\right]}^{\left[\mu_{1}, \ldots, \mu_{k}\right]}=\sum_{\sigma}(-1)^{\sigma} \delta_{\rho_{\sigma(1)}}^{\mu_{1}} \ldots \delta_{\rho_{\sigma(k)}}^{\mu_{k}}
$$

We will also use the notation $\epsilon \epsilon_{k, k}$ and $\delta_{k, k}$ as a multi-linear operator with $k$ free upper indices and $k$ free lower indices. If $k=0$, the symbol is a pure number $\epsilon \epsilon_{0,0}=N$ !.

We have the following identity if we introduce matrices

$$
\epsilon \epsilon_{N, N}\left(1, \ldots, 1, M_{1}, \ldots, M_{k}\right)=\epsilon \epsilon_{k, k}\left(M_{1}, \ldots, M_{k}\right)
$$

We can also write the above in terms of the $\delta_{k, k}$ symbol

$$
\begin{aligned}
\delta_{k, k}\left(M_{1}, \ldots, M_{k}\right) & =\delta_{\left[\nu_{1} \ldots \nu_{k}\right]}^{\left[\rho_{1}, \ldots, \rho_{k}\right]}\left(M_{1}\right)_{\rho_{1}}^{\nu_{1}} \ldots\left(M_{k}\right)_{\rho_{k}}^{\nu_{k}} \\
& =\sum_{\sigma}(-1)^{\sigma} \delta_{\rho_{\sigma(1)}}^{\mu_{1}} \ldots \delta_{\rho_{\sigma(k)}}^{\mu_{k}}\left(M_{1}\right)_{\rho_{1}}^{\nu_{1}} \ldots\left(M_{k}\right)_{\rho_{k}}^{\nu_{k}}
\end{aligned}
$$

Now, if we think of $\sigma$ as a permutation of the set $\{1, \ldots, k\}$, we can write the permutation in terms of a cycle representation $\hat{\sigma}=\left(i \sigma(i) \sigma^{2}(i) \ldots\right)\left(j \sigma(j) \sigma^{2}(j) \ldots\right) \ldots$ where $i \rightarrow \sigma(i) \rightarrow$ $\sigma^{2}(i)$ etc. The way the indices are contracted in the expression above A.9 give rise to traces, one for each parenthesis in the cycle representation of $\sigma$. The above then gives

$$
\delta_{k, k}\left(M_{1}, \ldots, M_{k}\right)=\sum_{\sigma}(-1)^{|\sigma|} \operatorname{tr}\left(M_{i} M_{\sigma(i)} M_{\sigma^{2}(i)} \ldots\right) \operatorname{tr}\left(M_{j} M_{\sigma(j)} \ldots\right) \ldots
$$

an alternating sum over all possible trace combinations of the matrices $M_{1}, \ldots, M_{k}$, determined by the permutations of $\{1, \ldots, k\}$.

In particular, if for each trace we associate a factor of $N$, then the above expression is a $1 / N$ expansion in terms of sums of traces, starting with

$$
\delta_{k, k}\left(M_{1}, \ldots, M_{k}\right)=N^{k}\left(\frac{\operatorname{tr}\left(M_{1}\right)}{N} \ldots \frac{\operatorname{tr}\left(M_{k}\right)}{N}\right)-N^{k-1} \sum_{i<j}\left(\frac{\operatorname{tr}\left(M_{i} M_{j}\right)}{N} \prod_{s \neq i, j} \frac{\operatorname{tr}\left(M_{s}\right)}{N}\right)+\ldots
$$


Now, we will consider the combinatorics of contracting four $\epsilon$ symbols. Let us consider $\epsilon \epsilon_{k, k} \epsilon \epsilon_{k, k}$ with all indices contracted between the two $\epsilon \epsilon$ symbols. This is equal to

$$
\begin{aligned}
\epsilon \epsilon_{k, k} \epsilon \epsilon_{k, k} & =\epsilon^{\mu_{1}, \ldots, \mu_{N}} \epsilon_{\mu_{1}, \ldots, \mu_{N-k}, \sigma_{N-k+1}, \ldots \sigma_{N}} \epsilon^{\sigma_{1}, \ldots, \sigma_{N}} \epsilon_{\sigma_{1}, \ldots, \sigma_{N-k}, \mu_{N-k+1}, \ldots \mu_{N}} \\
& =(N-k) !^{2} \delta_{k, k} \delta_{k, k} \\
& =N !(N-k) ! k !
\end{aligned}
$$

The last line follows from the fact that for the terms to contribute we need that the two sets $\left\{\mu_{1}, \ldots, \mu_{N-k}\right\}$ and $\left\{\sigma_{1}, \ldots, \sigma_{N-k}\right\}$ are identical, and that all terms contribute with the same sign. The number of ways of choosing indices for $\mu_{1}, \ldots, \mu_{N}$ is $N$ !. Now, the indices for $\sigma_{1}, \ldots, \sigma_{N-k}$ are fixed up to permutations, as well as $\sigma_{N-k-1}, \ldots, \sigma_{N-k}$. Each of these gives us an extra multiplicity of $k !(N-k)$ ! from permutations of the fixed sets. Notice the symmetry $k \rightarrow(N-k)$ of the final answer which is obvious from the index contractions. From the last line, we see that

$$
\delta_{k, k} \delta_{k, k}=k !^{2}\left(\begin{array}{c}
N \\
k
\end{array}\right)=k ! N^{k}(1-1 / N) \ldots(1-k / N)
$$

Similar to A.7, we can consider the $\delta \delta$ or $(\epsilon \epsilon)(\epsilon \epsilon)$ tensors as multilinear operators with various entries. We will be particularly interested in contracting the indices with matrices in such a way that all of the upper indices of one of the $(\epsilon \epsilon)$ are attached with the lower indices of the other $(\epsilon \epsilon)$ group.

The notation we will use for this is as follows

$$
\delta_{k, k} \delta_{\bar{k} \bar{k}}\left(M_{1}, \ldots, M_{k} ; \tilde{M}_{1}, \ldots \tilde{M}_{k}\right)=\delta_{\left[\rho_{1}, \ldots, \rho_{k}\right]}^{\left[\mu_{1}, \ldots, \mu_{k}\right]} \delta_{\left[\bar{\rho}_{1}, \ldots, \bar{\rho}_{k}\right]}^{\left[\bar{\mu}_{1}, \ldots, \bar{\mu}_{k}\right]}\left(M_{1}\right)_{\bar{\mu}_{1}}^{\rho_{1}} \ldots\left(M_{k}\right)_{\bar{\mu}_{k}}^{\rho_{k}}\left(\bar{M}_{1}\right)_{\mu_{1}}^{\bar{\rho}_{1}} \ldots\left(\bar{M}_{k}\right)_{\mu_{k}}^{\bar{\rho}_{k}}
$$

Now, we can use the identity A.6 to rewrite the above contraction as

$$
\begin{aligned}
\delta_{k, k} \delta_{\bar{k} \bar{k}}\left(M_{1}, \ldots, M_{k} ; \tilde{M}_{1}, \ldots \tilde{M}_{k}\right) & =\sum_{\sigma} \delta_{k, k}(-1)^{2|\sigma|} \delta_{k, k}\left(M_{1} \bar{M}_{\sigma(1)} \ldots M_{k} \bar{M}_{\sigma(k)}\right)( \\
& =\sum_{\sigma} \delta_{k, k}\left(M_{1} \bar{M}_{\sigma(1)}, \ldots, M_{k} \bar{M}_{\sigma(k)}\right)
\end{aligned}
$$

The fact that we get $(-1)^{2 \sigma}$ is that once we contract the upper index of $M_{i}$ with the lower index of $\bar{M}_{\sigma(i)}$ we need to take into account the permutation of the upper indices of $\bar{M}_{\sigma(i)}$ with respect to the lower indices of $M_{i}$. This permutation is $\sigma^{-1}$, so we get a factor of $\left((-1)^{|\sigma|}\right)^{2}$ from this extra reshuffling of indices. Now, we can use again identity A.10 to write the above as an alternating sum over all trace combinations of the composite matrices $M_{i} \bar{M}_{\sigma(i)}$, and over all possible permutations $\sigma$.

\section{References}

[1] J. M. Maldacena, "The large N limit of superconformal field theories and supergravity," Adv. Theor. Math. Phys. 2, 231 (1998) [Int. J. Theor. Phys. 38, 1113 (1999)] [arXiv:hep-th/9711200].

[2] G. 't Hooft, "Dimensional Reduction In Quantum Gravity," arXiv:gr-qc/9310026. 
[3] L. Susskind, "The World as a hologram," J. Math. Phys. 36, 6377 (1995) [arXiv:hep-th/9409089].

[4] G. 't Hooft, "A Planar Diagram Theory For Strong Interactions," Nucl. Phys. B 72, 461 (1974).

[5] J. McGreevy, L. Susskind and N. Toumbas, "Invasion of the giant gravitons from anti-de Sitter space," JHEP 0006, 008 (2000) [arXiv:hep-th/0003075].

[6] A. Hashimoto, S. Hirano and N. Itzhaki, "Large branes in AdS and their field theory dual," JHEP 0008, 051 (2000) [arXiv:hep-th/0008016].

[7] M. T. Grisaru, R. C. Myers and O. Tafjord, "SUSY and Goliath," JHEP 0008, 040 (2000) [arXiv:hep-th/0008015].

[8] E. Witten, "Anti-de Sitter space and holography," Adv. Theor. Math. Phys. 2, 253 (1998) [arXiv:hep-th/9802150].

[9] S. S. Gubser, I. R. Klebanov and A. M. Polyakov, "Gauge theory correlators from non-critical string theory," Phys. Lett. B 428, 105 (1998) [arXiv:hep-th/9802109].

[10] S. M. Lee, S. Minwalla, M. Rangamani and N. Seiberg, "Three-point functions of chiral operators in $\mathrm{D}=4, \mathrm{~N}=4 \mathrm{SYM}$ at large N," Adv. Theor. Math. Phys. 2, 697 (1998) [arXiv:hep-th/9806074].

[11] D. Berenstein, J. M. Maldacena and H. Nastase, "Strings in flat space and pp waves from N = 4 super Yang Mills," JHEP 0204, 013 (2002) [arXiv:hep-th/0202021].

[12] D. Kabat and W. I. Taylor, "Spherical membranes in matrix theory," Adv. Theor. Math. Phys. 2, 181 (1998) [arXiv:hep-th/9711078].

[13] S. J. Rey, "Gravitating M(atrix) Q-balls," arXiv:hep-th/9711081.

[14] D. Berenstein and R. Corrado, "Matrix theory on ALE spaces and wrapped membranes," Nucl. Phys. B 529, 225 (1998) [arXiv:hep-th/9803048].

[15] J. Maldacena, M. M. Sheikh-Jabbari and M. Van Raamsdonk, "Transverse fivebranes in matrix theory," JHEP 0301, 038 (2003) [arXiv:hep-th/0211139].

[16] N. w. Kim and J. Plefka, "On the spectrum of pp-wave matrix theory," Nucl. Phys. B 643, 31 (2002) [arXiv:hep-th/0207034].

[17] K. Dasgupta, M. M. Sheikh-Jabbari and M. Van Raamsdonk, "Protected multiplets of M-theory on a plane wave," JHEP 0209, 021 (2002) [arXiv:hep-th/0207050].

[18] J. M. Maldacena, G. W. Moore and N. Seiberg, "Geometrical interpretation of D-branes in gauged WZW models," JHEP 0107, 046 (2001) [arXiv:hep-th/0105038].

[19] S. S. Gubser, I. R. Klebanov and A. M. Polyakov, "A semi-classical limit of the gauge/string correspondence," Nucl. Phys. B 636, 99 (2002) [arXiv:hep-th/0204051].

[20] S. R. Das, A. Jevicki and S. D. Mathur, "Vibration modes of giant gravitons," Phys. Rev. D 63, 024013 (2001) [arXiv:hep-th/0009019].

[21] D. Berenstein, C. P. Herzog and I. R. Klebanov, "Baryon spectra and AdS/CFT correspondence," JHEP 0206, 047 (2002) [arXiv:hep-th/0202150].

[22] V. Balasubramanian, M. Berkooz, A. Naqvi and M. J. Strassler, "Giant gravitons in conformal field theory," JHEP 0204, 034 (2002) [arXiv:hep-th/0107119]. 
[23] V. Balasubramanian, M. x. Huang, T. S. Levi and A. Naqvi, "Open strings from $\mathrm{N}=4$ super Yang-Mills," JHEP 0208, 037 (2002) [arXiv:hep-th/0204196].

[24] S. Corley, A. Jevicki and S. Ramgoolam, "Exact correlators of giant gravitons from dual N = 4 SYM theory," Adv. Theor. Math. Phys. 5, 809 (2002) [arXiv:hep-th/0111222].

[25] O. Aharony, Y. E. Antebi, M. Berkooz and R. Fishman, "Holey sheets': Pfaffians and subdeterminants as D-brane operators in large N gauge theories," JHEP 0212, 069 (2002) [arXiv:hep-th/0211152].

[26] E. Witten, "Baryons In The 1/N Expansion," Nucl. Phys. B 160, 57 (1979).

[27] E. Witten, "Baryons and branes in anti de Sitter space," JHEP 9807, 006 (1998) [arXiv:hep-th/9805112].

[28] S. Gukov, M. Rangamani and E. Witten, "Dibaryons, strings, and branes in AdS orbifold models," JHEP 9812, 025 (1998) [arXiv:hep-th/9811048].

[29] S. S. Gubser and I. R. Klebanov, "Baryons and domain walls in an N = 1 superconformal gauge theory," Phys. Rev. D 58, 125025 (1998) [arXiv:hep-th/9808075].

[30] C. E. Beasley, "BPS branes from baryons," JHEP 0211, 015 (2002) [arXiv:hep-th/0207125].

[31] P. Ouyang, "Semiclassical quantization of giant gravitons," arXiv:hep-th/0212228.

[32] K. Intriligator and B. Wecht, "Baryon charges in 4D superconformal field theories and their AdS duals," arXiv:hep-th/0305046.

[33] C. P. Herzog and J. McKernan, "Dibaryon spectroscopy," arXiv:hep-th/0305048.

[34] C. Kristjansen, J. Plefka, G. W. Semenoff and M. Staudacher, "A new double-scaling limit of $\mathrm{N}=4$ super Yang-Mills theory and PP-wave strings," Nucl. Phys. B 643, 3 (2002) [arXiv:hep-th/0205033].

[35] D. Berenstein and H. Nastase, "On lightcone string field theory from super Yang-Mills and holography," arXiv:hep-th/0205048.

[36] N. R. Constable, D. Z. Freedman, M. Headrick, S. Minwalla, L. Motl, A. Postnikov and W. Skiba, "PP-wave string interactions from perturbative Yang-Mills theory," JHEP 0207, 017 (2002) [arXiv:hep-th/0205089].

[37] A. V. Ryzhov, "Quarter BPS operators in N = 4 SYM," JHEP 0111, 046 (2001) [arXiv:hep-th/0109064].

[38] E. D'Hoker, P. Heslop, P. Howe and A. V. Ryzhov, "Systematics of quarter BPS operators in N = 4 SYM," JHEP 0304, 038 (2003) [arXiv:hep-th/0301104].

[39] D. Z. Freedman, S. D. Mathur, A. Matusis and L. Rastelli, "Correlation functions in the CFT $(d) / \operatorname{AdS}(d+1)$ correspondence," Nucl. Phys. B 546, 96 (1999) [arXiv:hep-th/9804058].

[40] A. Mikhailov, "Giant gravitons from holomorphic surfaces," JHEP 0011, 027 (2000) [arXiv:hep-th/0010206]. 